\title{
Diagnosis of Malaria by Acridine Orange Fluorescent Microscopy in an Endemic Area of Venezuela
}

\author{
Irene Bosch ${ }^{*}$, Carmen Bracho, Hilda A Pérez $/^{+}$ \\ Laboratorio de Inmunoparasitología, Centro de Microbiología y Biología Celular, Instituto Venezolano de \\ Investigaciones Científicas (IVIC), Apdo 21827, Caracas 1020 A, Venezuela
}

Fluorescent (acridine orange) microscopical examination of capillary centrifuged blood (quantitative buffy coat [QBC®] analysis) and Giemsa stained thick blood smears (GTS) were compared for diagnosis of malaria in blood specimens from adults living in malaria transmission areas of the States of Bolivar and Amazonas in southeastern and south Venezuela, respectively. Of a total of 198 GTS examined, 95 subjects (48\%) showed parasitaemia. Among the 95 blood films with a positive GTS, 94 were judged positive by the QBC. However, positive QBC tubes were found in 29 out of 103 blood specimens with a negative GTS. Thus, relative to a GTS standard, the sensitivity and specificity of the QBC-test was $99.2 \%$ and $72 \%$, respectively. Young trophozoites of Plasmodium vivax and P. falciparum could not be distinguished with certainty. It is confirmed that the QBC offers many advantages compared with the standard diagnosis of malaria parasites, specifically in the speed of staining and ease of interpretation. However, in places where $\mathrm{P}$. falciparum and $\mathrm{P}$. vivax occur, species and stage differentiation should be confirmed with the GTS.

Key words: malaria - diagnosis - QBC test - Plasmodium vivax - Plasmodium falciparum

Malaria diagnosis is based on microscopic examination of Giemsa-stained thick blood smears (GTS). The method is specific (species and stage can be identified) and sensitive (4-10 parasites/ $\mu \mathrm{l}$ of blood can be determined). However, it is labor intensive and time consuming. Therefore, in endemic areas the efficiency of the GTS is compromised by the need to examine large number of blood samples, specially when patients have low density parasitaemias. Fluorochromes staining of malaria parasites, particularly with acridine orange in the QBC tube have been successfully applied to the diagnosis of malaria (Sodeman 1970, Spielman et al. 1988, Levine et al. 1989). This study was undertaken to compare the QBC method to the conventional examination of GTS for the diagnosis of malaria in blood specimens collected from individuals infected in various transmission areas of southeastern and south Venezuela.

\section{MATERIALS AND METHODS}

Patients - Three groups of patients were studied. Group 1 (preliminary study), consisted of 20 individuals who attended the outpatient malaria

This study received partial support from Grant PS/DRS/ RG/VEN66/126.

* Present address: Harvard University, School of Public Health, Dept. of Tropical Public Health, Boston, MA, U.S.A

+ Corresponding author

Received 8 August 1994

Accepted 31 October 1995 diagnosis laboratory in Caracas (División de Malariología y Saneamiento Ambiental, DMSA, Zone X, Caracas). Four negative controls (laboratory workers) were also included. Group 2 (field study), consisted of 141 subjects who had been living for a short period (2-12 months) in the gold mines of State of Bolivar (southeastern Venezuela) and who presented to the outpatient malaria diagnosis laboratory of Ciudad Bolivar, State of Bolivar, (DMSA, Zone III). Group 3 (field study), consisted of 37 indigenous children aged 2-11 years of the Guahibo ethno, living in "Alcabala de Guahibos", State of Amazonas. The research team visited the indigenous community to search for malaria cases. These children had been receiving a weekly suppressive dose of chloroquine during the last three weeks, and all of them were asymptomatic at the time of blood collection.

Collection and examination of blood samples - Patients were submitted to one finger-stick with a standard lancet, from which a thick and thin blood smears were prepared. Fifty five microliters of blood were drawn into a single QBC@ capillary tube (Becton-Dickinson, Inc., Franklin Lakes, NJ) from the same finger-stick. Samples in the QBC tube were processed according to manufacturer instructions. Blood specimens of Group 1 were centrifuged and examined within 1-2 $\mathrm{hr}$ of collection in the central laboratory at IVIC. Blood specimens of Groups 2 and 3 were centrifuged within 2-4 hr of collection and examined in the malaria diagnosis unit either in Ciudad Bolivar or Pto Ayacucho. Samples of patients from Groups 
1 and 2 were examined using a x10 ocular lens and a $x 50$ objective immersion lens. The microscope was a binocular fluorescent (Zeiss, Standard model, Germany) equipped with an incident light fluorescent source. Sample of Group 3 were examined using a standard light microscope (Lavobal, Venezuela) to which a fluorescent system (Becton-Dickinson, USA) was assembled. Microscopic examination of GTS was carried out separately by two well trained microscopists from the DMSA. Two hundred fields were examined at X1000 magnification. Two hundred fields were estimated to contain $0.33 \mu \mathrm{l}$ of blood. The sensitivity was calculated as $100 \mathrm{x}$ the number of specimens positive by both QBC and GTS divided by the total number of positives GTS. The specificity was calculated as $100 \mathrm{x}$ the number of specimens negative by both GTS and QBC divided by the total number of negative GTS (Rickman et al. 1989).

\section{RESULTS}

Malaria diagnosis by the QBC-test - Group 1: of the 20 subjects from Group 1, 14 were found positive by the GTS, ten had $P$. vivax and four had $P$. falciparum. Parasites were detected by acridine orange in all of these subjects. No false positives were diagnosed in the four negative controls or in the two convalescent patients who had received radical treatment for $P$. vivax malaria two weeks before blood examination. This pilot experiment allowed us to become familiar with the stratification of parasites in the QBC tube, specially that of mature schizont of $P$. vivax which tends to distribute in the granulocyte layer. In patients with $P$. falciparum, parasitaemias ranged from 450-2000 parasites/ $\mu$ l of blood. Those infected with $P$. vivax had parasitaemias ranging from 200-1500 parasites/ $\mu 1$. Group 2: of a total of 141 blood smears screened by the GTS, 70 showed malaria parasites; 69 out of 70 patients with a positive GTS were positive in the QBC test. Eleven out of the 37 indigenous children from Group 3 which exhibited a positive GTS also gave a positive result for the QBC test. Sensitivity of the QBC test as compared to GTS was $99 \%$ and $100 \%$ for Groups 2 and 3, respectively (Table I). Comparison of the results obtained by the QBC test and the GTS indicated that $49 \%(69 / 141)$ of the patients in Group 2 had a positive malaria diagnosis by both tests (QBC+/GTS+), 32\% (46/141) failed to show malaria parasites (QBC-/GTS-); $18 \%(26 / 141)$ showed a positive QBC tube but a negative GTS (QBC+/GTS-) and one patient (1\%) gave a positive GTS but a negative $\mathrm{QBC}$ tube (QBC-/GTS+) (Table II). A similar comparison for patients of Group 3 indicated that 30\% (11/ 37) of children examined were QBC+/GTS+; $59 \%$
(22/37) resulted negative by both tests (QBC-/ GTS-) and 11\% (4/37) had a positive QBC tube but a negative GTS (Table II).

In order to compare the sensitivity of the QBC for the diagnosis of vivax and falciparum malaria results from Groups 1 to 3 were pooled. Ninety four out 95 samples with a positive slide for malaria parasites gave a positive QBC tube. Hence, the sensitivity of the QBC test for the over all sample showing a positive GTS was 99\% (94/ 95). Morphological identification of the infecting malaria parasites by examination of the thin smears indicated that 63 patients had $P$. vivax malaria and 32 were infected vith $P$. falciparum. Sensitivity of the QBC test was $100 \%$ for P. vivax and $97 \%$ for $P$. falciparum. Parasitaemias ranged from 200-1500 parasites/ $\mu$ for $P$. vivax and from 1002000 parasites/ $\mu$ for $P$. falciparum (Table III). It should be pointed out that the QBC-test showed direct evidence of infection in 29 out of 103 blood specimens with a negative GTS. Further examination of slides (200 fields) with a positive QBC tube and a negative GTS failed to detect the presence of parasites. Hence, relative to a GTS standard, the specificity of the QBC-test was 72\%.

\section{TABLE I}

Sensitivity of QBC test in a field study of patients infected in transmission areas of southeastern (State of Bolivar) and south (State of Amazonas) Venezuela

\begin{tabular}{ccc}
\hline Group $^{a}$ & $\begin{array}{c}\text { Number of subjects } \\
\text { studied }\end{array}$ & $\begin{array}{c}\text { Number of patients } \\
\text { QBC+/GTS+ }\end{array}$ \\
\hline 2 & 141 & $69 / 70(99 \%)^{c}$ \\
3 & 37 & $11 / 11(100 \%)$ \\
\hline
\end{tabular}

a: Group 2, 141 miners of State of Bolivar; Group 3, 37 indigenous children of State of Amazonas

$b: \mathrm{QBC}=$ quantitative buffy coat $(\mathrm{QBC} \AA)$ analysis and GTS $=$ Giemsa stained thick blood smears

$c$ : number in bracets indicate sensitivity for the QBCtest relation to GTS.

\section{TABLE II}

Comparison of the $\mathrm{QBC}^{a}$ test and $\mathrm{GTS}^{b}$ to determine prevalence $(\%)$ of malaria in a field study of individuals living in endemic areas of Venezuela

\begin{tabular}{cccccc}
\hline \multirow{2}{\text{Group}^{c}}{} & QBC+ & $\frac{\text { QBC- }}{\text { GTS+ }}$ & GTS- & $\frac{\text { QBC+ }}{\text { GTS- }}$ & $\frac{\text { QBC- }}{\text { GTS+ }}$ \\
\hline 2 & 49 & 32 & 18 & 1 \\
3 & 30 & 59 & 11 & - \\
\hline
\end{tabular}

$a, b: \mathrm{QBC}=$ quantitative buffy coat $(\mathrm{QBC}()$ analysis and GTS = Giemsa stained thick blood smears $c$ : Group 2: 141 miners (State of Bolivar); Group 3: 37 indigenous children living in State of Amazonas. 


\section{TABLE III}

Sensitivity of the $\mathrm{QBC}^{a}$ test as compared to the $\mathrm{GTS}^{b}$ in patients with Plasmodium falciparum or $P$. vivax malaria

\begin{tabular}{lcc}
\hline $\begin{array}{l}\text { Patients } \\
\text { infected with }^{c}\end{array}$ & $\begin{array}{c}\text { Sensitivity }^{d}(\%) \text { of } \\
\text { the } \mathrm{QBC} \text { test }\end{array}$ & $\begin{array}{c}\text { Range of parasitaemia } \\
\text { (parasites } / \mu \mathrm{l})\end{array}$ \\
\hline $\begin{array}{l}\text { P. vivax }(\mathrm{n}=63) \\
\text { p. falciparum }(\mathrm{n}=32)\end{array}$ & 100 & $200-1500$ \\
\hline
\end{tabular}

$a, b: \mathrm{QBC}=$ quantitative buffy coat $(\mathrm{QBC} \AA)$ analysis and GTS = Giemsa stained thick blood smears

$c$ : identification of parasite species was done by microscopic examination of Giemsa stained thin smears $d$ : the test sensitivity defined as the percentage of positive GTS that were positive by the QBC test.

\section{DISCUSSION}

In this study we evaluated the ability of the acridine orange staining of centrifuged parasites in microhematocrit tubes ( $\mathrm{QBC}\left({ }^{\circ}\right)$ to detect malaria under field conditions. Malaria patients were either symptomatic subjects who had visited the gold mines of State of Bolivar (Groups 1 and 2) or asymptomatic indigenous children living in an endemic village of State of Amazonas (Group 3). Results indicated that $99 \%$ of patients with a positive GTS gave a positive result with the QBC test. However, relative to GTS the specificity of the QBC for the over all sample was $72 \%$. Analysis of history data indicated that $100 \%$ of individuals in Group 2 with a negative GTS but a positive QBC had been living during the last 2 to 12 months in malaria endemic areas. Sixty one per cent (16/26) of them complained of a febrile episode which had occurred three to five days before blood examination and 38\% (10/26) referred to antimalarial drugs intake during the last three weeks. A similar situation was observed with the blood samples of the indigenous children. There were four additional QBC positive specimens, all of them from children who had received prophylactic antimalarial drug treatment during the last three weeks.

Sensitivity and specificity of the QBC malaria diagnosis compared to GTS ranging from $75 \%$ to $99.6 \%$ and $82 \%$ to $98.4 \%$, respectively, have been reported (Rickman et al. 1989, Pornsilapatip et al. 1990, Wongsrichanalai et al. 1991, Baird et al. 1992, Cabezos \& Bada 1993, Gay et al. 1994, Benito et al. 1994). Difficulties to follow-up patients with a positive QBC test and a negative GTS seem to complicate determination of QBC specificity (Rickman et al. 1989, Porsilapatip et al. 1990, Long et al. 1994). However, conversion from negative to positive GTS in individuals with an early positive QBC have been observed (Rickman et al. 1989, Porsilapatip et al. 1990, Benito et al. 1994). In a recent study Long et al. (1994) provided evidence of the limitations of the GTS for the diagnosis of malaria in patients with very low parasitaemias $(<10$ parasites/ $\mu \mathrm{l})$. The authors showed that in 49 subjects experimentally infected with $P$. falciparum diagnosis was earlier accomplished by the QBC than by the GTS in 23 (47\%) individuals. It seems therefore, that at least part of the controversy on the specificity of the QBC reflects the contradiction of comparing a new diagnostic method with an old reference test that could be less sensitive.

In our experience diagnosis of malaria by the acridine orange staining of centrifuged parasites in microhematocrite tubes was considerably more rapid and easy to learn than GTS and the availability of the portable fiber optic paralens made it possible to read and interpret results in any peripheral laboratory of the national network for the diagnosis and treatment of malaria. Therefore, the QBC seems quite useful to the rapid identification of malaria infected subjects. However, there are several drawbacks to be considered. One is the identification of Plasmodium species. Ring stages of $P$. falciparum and $P$. vivax are difficult to distinguish by the $\mathrm{QBC}$. This problem is particularly important in endemic areas where P. falciparum coexists with $P$. vivax. Under that circumstances individuals with a positive $\mathrm{QBC}$ should be further evaluated by a Giemsa stained blood smear in order to confirm infecting species of malaria parasites and to administrate the appropriate drug treatment.

\section{ACKNOWLEDGMENTS}

To Dr J Perrone (Becton-Dickinson) for supplying the QBC System (tubes, centrifuge, lens and portable U.V. light source) and the laboratory staffs of DMSA, Zone III (Ciudad Bolivar, Edo. Bolivar) Zone X (Caracas) and Zone XX (Pto Ayacucho, Edo. Amazonas).

\section{REFERENCES}

Baird K, Purnomo, Jones TR 1992. Diagnosis of malaria in the field by fluorescent microscopy of QBC@ capillary tubes. Trans $R$ Soc Trop Med Hyg 86: 3-5.

Benito A, Roche J, Molina R, Amela C, Alvar J 1994. Application and evaluation of QBC malaria diagnosis in a holoendemic area. Appl Parasitol 35: 266-272.

Cabezos J, Bada JL 1993. The diagnosis of malaria by the thick film and the QBC: a comparative study of both techniques. Med Clin Barc 101: 91-94.

Gay F, Reaore B, Zanoni J, Danis M, Gentillini M 1994. Evaluation of the QBC system for the diagnosis of malaria. Santé 4: 289-297.

Levine RA, Wardlaw SC, Patton, CL 1989. Detection of haemoparasites using quantitative buffy coat analysis tubes. Parasitol Today 5: 132-134. 
Long G, Jones T, Rickman LS, Fries L, Egan J, Wellde B, Hoffman S 1994. Acridine orange diagnosis of Plasmodium falciparum: evaluation after experimental infection. Am J Trop Med Hyg 51: 613-616.

Pornsilapatip J, Namsiripongpun V, Wilde H, Hanvanich $\mathrm{M}$, Chutivongse S 1990. Detection of plasmodia in acridine orange stained capillary tubes (the QBC system). SE Asian J Trop Med Publ Health 21: 534540.

Rickman LS, Long GW, Oberts R, Caraban A, Sangalang R, Smith JI, Chulay JD, Hoffman SL 1989. Rapid diagnosis of malaria by acridine orange staining of centrifuged parasites. Lancet $i$ : 68-71
Sodeman TM 1970. The use of fluorochromes for the detection of malaria parasites. Am J Trop Med Hyg 19: 40-42.

Spielman A, Perrone JB, Teklahaimanot A, Balcha F, Wardlaw SC, Levine RA 1988. Malaria diagnosis by direct observation of centrifuged samples of blood. Am J Trop Med Hyg 39: 337-342.

Wongsrichanalai C, Pornilapatip J, Namsiripongpun H, Webster H, Luccini A, Pansadang P, Wilde H, Prasittisuk M 1991. Acridine orange fluorescent microscopy and the detection of malaria in populations with low-density parasitemia. Am J Trop Med Hyg 44: 17-20. 